ARTICLE

https://doi.org/10.1038/s41467-019-11264-z

\title{
Real-time optical and electronic sensing with a $\beta$-amino enone linked, triazine-containing 2D covalent organic framework
}

\author{
Ranjit Kulkarni (10) 1,2, Yu Noda (1) 1,2, Deepak Kumar Barange (10 2, Yaroslav S. Kochergin 1,2,3, Pengbo Lyu (1) 4 , \\ Barbora Balcarova ${ }^{2}$, Petr Nachtigall ${ }^{4}$ \& Michael J. Bojdys (i) ${ }^{1,2}$
}

Fully-aromatic, two-dimensional covalent organic frameworks (2D COFs) are hailed as candidates for electronic and optical devices, yet to-date few applications emerged that make genuine use of their rational, predictive design principles and permanent pore structure. Here, we present a 2D COF made up of chemoresistant $\beta$-amino enone bridges and Lewis-basic triazine moieties that exhibits a dramatic real-time response in the visible spectrum and an increase in bulk conductivity by two orders of magnitude to a chemical trigger - corrosive $\mathrm{HCl}$ vapours. The optical and electronic response is fully reversible using a chemical switch $\left(\mathrm{NH}_{3}\right.$ vapours) or physical triggers (temperature or vacuum). These findings demonstrate a useful application of fully-aromatic 2D COFs as real-time responsive chemosensors and switches.

\footnotetext{
${ }^{1}$ Department of Chemistry, Humboldt-Universität zu Berlin, Brook-Taylor-Str. 2, 12489 Berlin, Germany. ${ }^{2}$ Institute of Organic Chemistry and Biochemistry of the CAS, Flemingovo nám. 2, Prague 166 10, Czech Republic. ${ }^{3}$ Department of Organic Chemistry, Charles University in Prague, Hlavova 8,12840 Prague, Czech Republic. ${ }^{4}$ Department of Physical and Macromolecular Chemistry, Charles University in Prague, Hlavova 8, Prague 128 40, Czech Republic. Correspondence and requests for materials should be addressed to M.J.B. (email: m.j.bojdys.02@cantab.net)
} 
O rganic semiconductor materials find use in sensing applications $^{1}$, opto-electronic devices ${ }^{2,3}$, field-effect transistors ${ }^{4}$, light-emitting diodes (LEDs) ${ }^{5}$, photovoltaic and solar cells ${ }^{6}$. The efficiency of these devices largely depends on the band gap position and energy and charge-carrier mobilities. Band gap energy and charge transport depend not only on the degree of extended $\pi$-conjugation and planarity of the conjugated sub-units, but also on their chemical structure ${ }^{7,8}$. In contrast to many post-synthetic strategies for enhancing charge-carrier mobilities and band gap tuning, organic framework materials, such as covalent organic frameworks (COFs), offer predictive synthetic tools to design structure and properties from the molecular building block to the extended macroscopic material. Thus, rational incorporation of electron-donating and withdrawing moieties into covalent organic frameworks can potentially improve their performance in electronic applications ${ }^{9}$.

Covalent organic frameworks (COFs) are a well-established class of porous, crystalline networks made up from light elements $(\mathrm{H}, \mathrm{B}, \mathrm{C}, \mathrm{N} \text { and } \mathrm{O})^{10,11}$. They are comprised of $2 \mathrm{D}$ covalently linked sheets that stack via $\pi-\pi$ interactions. Early COFs are linked by boronate esters and boroxins that are easily hydrolysed and oxidised ${ }^{12-15}$, and by more stable imines ${ }^{16}$. Their use in sensing applications and electrochemical devices has been hampered by the poor stability of these early COFs. We recently reported triazine $\left(\mathrm{C}_{3} \mathrm{~N}_{3}\right)$-based graphdiyene frameworks that are not only crystalline but also stable with respect to a wide range of chemicals and temperatures and that show interesting photocatalytic and electronic effects as well as structural polymorphism ${ }^{17,18}$

In this study, we condense a $C_{2}$ symmetric keto-enol, (1,4phenylene)bis(3-hydroxyprop-2-en-1-one) (PBHP), and a $C_{3}$ symmetric tri-amine, 1,3,5-tris-(4-aminophenyl) triazine (TAPT), into a 2D-layered COF linked by $\beta$-amino enones (Fig. 1), in analogy to hydrolytically stable $\beta$-keto-enamine linkers obtained from $C_{3}$ symmetric keto-enols and $C_{2}$ symmetric amines ${ }^{19}$. This material is the first COF to show a rapid sensing response in the visible spectrum for volatile acid vapours, which we attribute to protonation of its Lewis-basic triazine-moieties accompanying with enhanced conductivity by $\sim 170$-fold in the protonated state. These effects take advantage of the open pore structure of the $\mathrm{COF}$ as well as its modular make-up that allows incorporation of Lewis-acidic and basic sites and, hence, enables intrinsic donoracceptor behaviour that enhances band gap modularity and charge-carrier mobility. Intriguingly, in addition to the polycrystalline powder morphology observed for most COFs, PBHPTAPT COF grows in macroscopic, crystalline films at all flat interfaces of the reactor setup analogous to the surface-templated syntheses we used successfully for other layered 2D materials ${ }^{20,21}$. This unlocks applications in large-scale sensors and electronic devices, which we explore herein.

\section{Results}

$\boldsymbol{\beta}$-amino enone linked, triazine-containing $2 \mathrm{D}$ covalent organic framework. The triazine-containing PBHP-TAPT COF is an extension of the family of $2 \mathrm{D}$ COFs obtained via Michael addition-elimination ${ }^{22}$. The triazine-moiety in particular is an intriguing building block for frameworks with electronic and sensing end-applications, since its electron-accepting capacity is enhanced in the excited, protonated state ${ }^{23}$. Formation of the $\beta$-amino enone bridge is accompanied by irreversible tautomerization $^{24}$, which is likely to produce defects and, hence, a disordered framework under standard conditions. Hence, we condensed PBHP and TAPT in a 3:2 molar ratio under solvothermal conditions in a sealed glass ampule to allow for dynamic bond formation close to equilibrium (Fig. 1). PBHP-
TAPT COF precipitates as an orange solid and grows on all reactor interfaces as an orange film with an overall yield of $79 \%$ (Supplementary Fig. 3, for detailed procedures). We optimized the synthetic protocol by monitoring the intensity of crystalline peaks in powder X-ray diffraction (PXRD) measurements with varying ratios of the solvent mixture, mesitylene and dioxane $e^{25,26}$. The highest intensity of characteristic peaks was obtained for a 9:1 volume ratio of mesitylene to dioxane (Supplementary Fig. 4).

Combustion elemental analysis of PBHP-TAPT COF shows a composition of $\mathrm{C}, \mathrm{H}$ and $\mathrm{N}$ at $71.3 \%, 4.4 \%$ and $12.5 \%$, respectively, which is reasonably close to the theoretical values of $74.6 \%, 4.3 \%$ and $13.3 \%$ (Supplementary Table 1). Thermogravimetric analysis (TGA) under air and nitrogen shows that PBHP-TAPT COF is stable up to $\sim 420^{\circ} \mathrm{C}$. Since no appreciable weight loss is observed before the onset of decomposition, we conclude that the framework is virtually free of trapped guest molecules (Supplementary Fig. 5). The FT-IR spectra of PBHPTAPT COF powder show the disappearance of $\mathrm{N}-\mathrm{H}$ stretches and the emergence $\mathrm{C}-\mathrm{N}$ and $\mathrm{C}=\mathrm{N}$ vibrations at 1223 and $1628 \mathrm{~cm}^{-1}$ (Fig. 2a, Supplementary Fig. 22) ${ }^{22,27}$. Likewise, O-H vibrations seen for the keto-enol PBHP between 2800 and $2900 \mathrm{~cm}^{-1}$ disappear completely after condensation. Importantly, characteristic signals of the TAPT building blocks stemming from the triazine stretching mode $\left(1501 \mathrm{~cm}^{-1}\right)$, breathing mode $\left(1366 \mathrm{~cm}^{-1}\right)$ and out-of-plane ring bending $\left(809 \mathrm{~cm}^{-1}\right)$ are retained ${ }^{18} \cdot{ }^{13} \mathrm{C}$ cross-polarization magic angle spinning (CP-MAS) solid-state NMR spectroscopy confirms the formation of $\beta$-amino enone bridges showing characteristic signals for the carbonyl carbons at $189 \mathrm{ppm}$, the $\beta$-carbon at $142 \mathrm{ppm}$, and the $\alpha$-carbon at $95 \mathrm{ppm}$ (Fig. $2 \mathrm{~b})^{22}$. Triazine ring carbon environments are assigned to the peak at $169 \mathrm{ppm}^{21}$.

The PXRD pattern of PBHP-TAPT COF confirms the formation of a 2D-layered hexagonal network. Indexing and Le Bail refinement readily matches the observed profile using a hexagonal unit cell $P-6$ (no. 174) $\left(R_{\mathrm{wp}}=1.10 \%\right)$. To account for the stacking disorder we modelled conceivable stacking modes using density functional theory (DFT). Three arrangements typical for 2D COFs were considered: AA-eclipsed, AA'-serrated and $\mathrm{AB}$-staggered. Calculations show further that the $\mathrm{AA}$ and AA' packing modes are significantly more stable than the AB-staggered structure (Supplementary Table 2). Indeed, the AA packing mode gave the best match with the observed diffraction profile (Fig. 2c, d, and Supplementary Figs. 6 and 7), and it was subsequently used for structure refinement using Pawley methods with a low residual $\left(R_{\mathrm{wp}}=3.91 \%\right)$. PBHP-TAPT COF has a dominant diffraction peak at $2.13^{\circ}$ as well as smaller peaks at $3.8^{\circ}, 4.5^{\circ}$ and $6.1^{\circ}$ and a broad peak at $25.5^{\circ} 2 \theta(\mathrm{Cu} \mathrm{Ka}$ $\lambda=0.15406 \mathrm{~nm})$. These peaks correspond to the (100), (110), (200), (210) in-plane reflections as well as the (001) stacking reflection. In-plane distances between neighbouring pore channels are $4.1 \mathrm{~nm}$, hence, the expected pore diameter is $\sim 2.0 \mathrm{~nm}$, and the average inter-layer distance is $0.34 \mathrm{~nm}$, which is typical for van der Waals stacked 2D materials.

Scanning electron microscopy (SEM) shows that the bulk of PBHP-TAPT COF powder consists of spherical particles with diameters ranging between 5 and $20 \mu \mathrm{m}$, which is indicative of emulsion polymerisations (Supplementary Fig. 8). However, the powder can be mechanically broken up using ultrasonication, and reveal flat terraces in atomic force microscopy (AFM) measurements with average flake thicknesses of $9.9 \mathrm{~nm}$ (Supplementary Fig. 9). High-resolution transmission electron microscopy (HRTEM) confirms the inter-layer spacing as $3.6 \AA$ (Supplementary Fig. 10).

Nitrogen sorption isotherms of PBHP-TAPT COF show Type I behaviour, indicative of pores, with a guest-accessible Brunauer-Emmett-Teller surface area $\left(S_{\mathrm{BET}}\right)$ of $176 \mathrm{~m}^{2} \mathrm{~g}^{-1}$ 


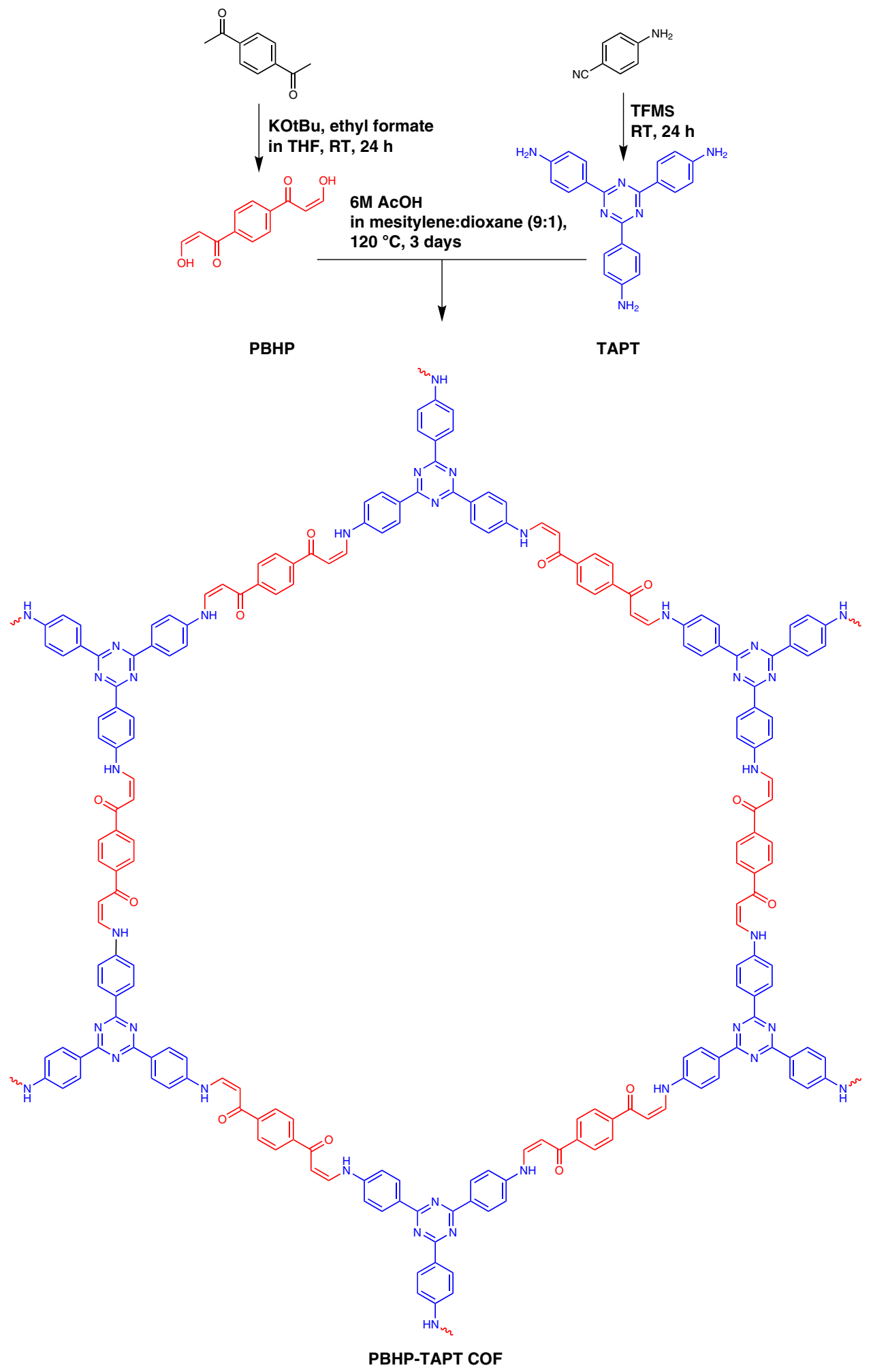

Fig. 1 Reaction scheme for PBHP-TAPT COF and its constituent building blocks

(Supplementary Fig. 11d). Pore size distribution was calculated using quenched solid-state functional theory (QSDFT) (Supplementary Fig. 11e), and the largest contribution to pore volume stems from pores of diameter $3.9 \mathrm{~nm}$, which is close to the pore channel diameter of the refined structure of $3.6 \mathrm{~nm}$ (Supplementary Fig. 11a). Note, that the observed surface area is far from the expected Connolly surface area of $2399 \mathrm{~m}^{2} \mathrm{~g}^{-1}$. Since no evidence of stoichiometric incorporation of guest molecules was found by elemental analysis, ${ }^{13} \mathrm{C}$ CP-MAS solid-state NMR, and TGA, we conclude that stacking defects or interdigitation of neighbouring layers must account for the apparent loss in accessible surface area. Indeed, the calculated energy differences between stacking 

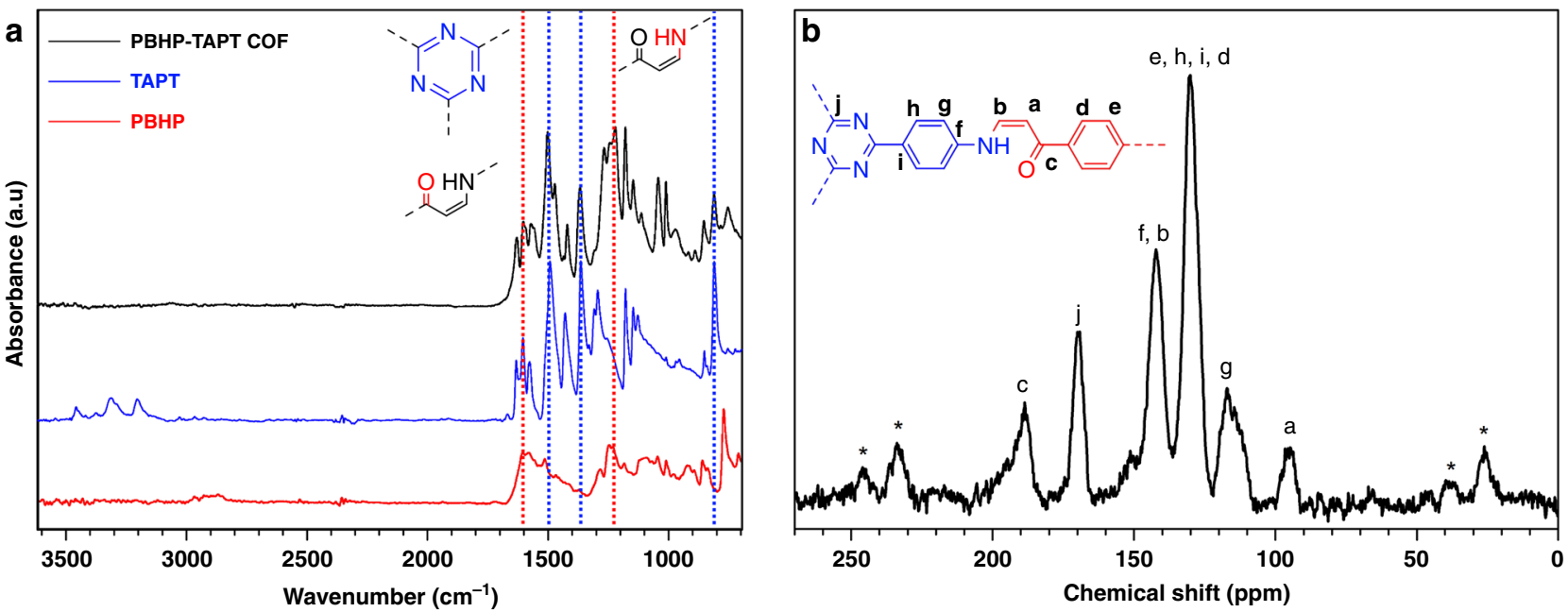

C

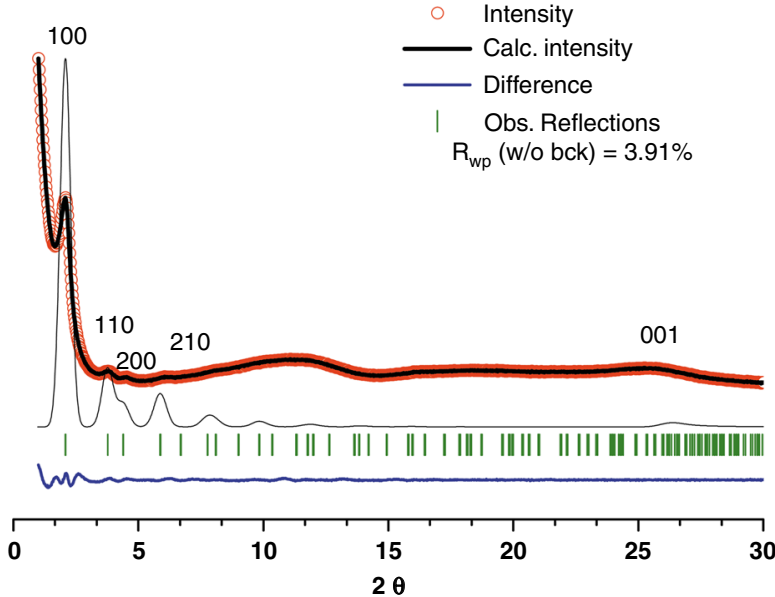

d

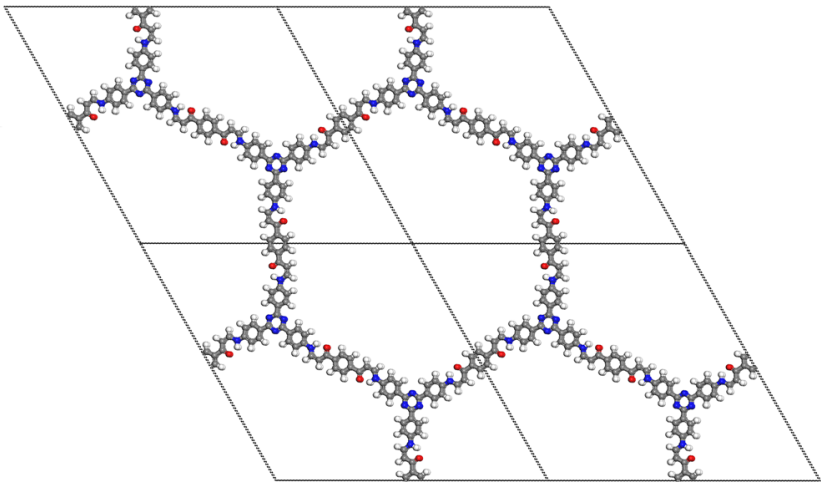

Fig. 2 Physical characterization of PBHP-TAPT COF. a Comparison of the Fourier-transform infrared (FT-IR) spectra of PBHP-TAPT COF (in black) with its building blocks TAPT (in blue) and the PBHP (in red). $\mathbf{b}^{13} \mathrm{C}$ cross-polarisation (CP) magic angle spinning (MAS) NMR (MAS rate of $13 \mathrm{kHz}$ ) of PBHP-TAPT COF. Spinning sidebands are marked with asterisks $\left(^{\star}\right)$. c Experimental (red circles), Pawley refined (thick black line), and predicted (thin black) PXRD patterns of PBHP-TAPT COF. Bragg peak positions (green), and the difference plot (experimental minus refined; blue). d $2 \times 2$ unit cells ( $a=44.2785 \AA$, $c=3.3767 \AA$, space group 174) of PBHP-TAPT COF with the principal unit cell (black dashes). Carbon (grey), hydrogen (white), nitrogen (blue), oxygen (red) atoms are represented as spheres

modes vary between 0.1 and $4.3 \mathrm{eV}$ (Supplementary Table 2), hence, there is a high likelihood that even a small number of stacking defects occlude most of the theoretically accessible surface area.

The modest surface area of PBHP-TAPT COF was, however, achieved without the need for elaborate post-synthetic activation techniques such as supercritical $\mathrm{CO}_{2}$ or solvent exchange which find their way into COF literature (Supplementary Fig. $11 \mathrm{~d})^{22,28}$.

The orange colour of PBHP-TAPT COF stems from an absorption edge at $525 \mathrm{~nm}$ according to solid-state UV/Vis diffuse reflectance spectroscopy (Supplementary Fig. 12), and according to the Kubelka-Munk function it corresponds to a direct band gap of $2.32 \mathrm{eV}$ and an indirect band gap of $2.06 \mathrm{eV}$. Solid-state photoluminescence (PL) spectrum shows an emission maximum at $590 \mathrm{~nm}(2.10 \mathrm{eV})$, which is closer to the calculated indirect band gap value (Supplementary Fig. 13), suggesting that PBHP-TAPT COF is an indirect band gap semiconductor. This combination of an optical band gap in the visible spectrum, guestaccessible pore volume, a strong covalent backbone and the presence of conjugated Lewis-basic moieties in the form of triazine cores make PBHP-TAPT COF an excellent candidate material for sensing of volatile, corrosive gases.

Real-time opto-electronic response. In a first set of experiments, we tested the response of PBHP-TAPT COF to $\mathrm{HCl}$ vapours. The as-received powder showed a rapid colour change from orange to red (shift of the UV/Vis absorption edge to $630 \mathrm{~nm}$ ) within seconds of exposure to $\mathrm{HCl}$ vapours at $1 \mathrm{~atm}$ and $\mathrm{RT}$. This coloration is fully reversible when the $\mathrm{HCl}$-treated sample is exposed to $\mathrm{NH}_{3}$ vapours at the same conditions (shift of the UV/Vis absorption edge back to $525 \mathrm{~nm}$ ) (Supplementary Fig. 14 and Supplementary Movie 1). The reversibility of the PBHP-TAPT COF was investigated by cycling the reaction with $\mathrm{HCl}$ and $\mathrm{NH}_{3}$ probes. The cycling test showed the retention of sensing ability of PBHP-TAPT COF, in five consecutive $\mathrm{HCl}-\mathrm{NH}_{3}$ exposure cycles (Supplementary Fig. 15a). Structural integrity of PBHP-TAPT $\mathrm{COF}$ after cycles with $\mathrm{HCl} / \mathrm{NH}_{3}$ gas exposure was confirmed with Fourier-transform infrared (FT-IR) spectra measurements (Supplementary Fig. 15b). The cycled samples showed no marked changes in the skeleton of PBHP-TAPT COF upon exposure to $\mathrm{HCl} / \mathrm{NH}_{3}$ gas. 
Furthermore, PBHP-TAPT COF, was highly sensitive to low concentration of $\mathrm{HCl}$ gas down to $20-50 \mathrm{ppm}$, detectable by UVVis. and the higher threshold was evaluated at around $3000 \mathrm{ppm}$ (Supplementary Fig. 16). This rapid, colour change offers an instant advantage over other optical sensors for corrosive gases where good reversibility and real-time response are lacking ${ }^{29,30}$.

In the protonated, excited state the direct and indirect band gaps of PBHP-TAPT COF decrease to 2.00 and $1.78 \mathrm{eV}$, respectively-a change by $\sim 0.3 \mathrm{eV}$. PBHP-TAPT COF remains structurally stable throughout the exposure to $\mathrm{HCl}$ and $\mathrm{NH}_{3}$ vapours as seen in PXRD profiles (Supplementary Fig. 17). Intriguingly, the protonated, excited framework can also be fully regenerated by thermal treatment $\left(>120^{\circ} \mathrm{C}\right.$ for $\left.60 \mathrm{~min}\right)$ or in vacuum. Structural integrity of PBHP-TAPT COF after physical and chemical regeneration was confirmed with Fourier-transform infrared (FT-IR) spectra and solid-state UV-Vis measurements (Supplementary Fig. 26).

The trend in colour change observed in UV/Vis is mirrored in PL spectroscopy. Not only does the emission maximum shift to $660 \mathrm{~nm}(1.84 \mathrm{eV})$ upon $\mathrm{HCl}$ exposure, but the overall fluorescence is also quenched. Calculations suggest that the triazine ring has a larger hyperpolarisability and more electron withdrawing character than the analogous carbon only benzene core ${ }^{31}$. We therefore conclude that the observed fluorescence quenching of protonated PBHP-TAPT COF is a consequence of electron donation into the protonated triazine ring, an effect that we have previously observed for strong donor-acceptor interactions in triazine-containing, conjugated microporous polymers ${ }^{31}$ and for molecular compounds ${ }^{32}$.

PBHP-TAPT COF has in principle three potential sites that can act as Lewis-bases (Supplementary Fig. 18), so to verify the site at which protonation occurs, we synthesised two sub-units of the COF backbone (Supplementary Fig. 18), keto-enamine (KE) and tri-phenyl triazine (TPT) and two model compounds 1,3,5-tris(4-aminophenyl)benzene (TAP) and 1,3,5-tris-(4-aminophenyl) triazine (TAPT) (Supplementary Fig. 19). These model compounds were exposed to $\mathrm{HCl}$ vapours at the same condition as the bulk powder and UV/Vis spectra were recorded. As expected, only the protonated, triazine-containing model compounds showed a red shift of the absorption edge, while the KE core did not (Supplementary Fig. 18b and 18c). The solid-state ${ }^{13} \mathrm{C} \mathrm{CP}$-MAS NMR spectrum of the protonated PBHP-TAPT COF has the best correlation with the triazine ring-nitrogen as the preferred protonation site (Supplementary Figs. 27 and 28). A computational investigation of the protonation of PBHP-TAPT COF shows that a protonated triazine linker is preferred by $70 \mathrm{~kJ} \mathrm{~mol}^{-1}$ over a protonated keto-enamine bridge (Supplementary Figs. 20 and 21). The ring protonation of triazine core affecting the red shift have already been reported for some oligomeric/polymeric systems and our observation also complies with it ${ }^{23,31,33,34}$. Further evidence that the site of protonation is indeed the triazine core comes from FT-IR spectroscopy (Supplementary Fig. 22). None of the signals belonging to the $\beta$-amino enone bridges or the aromatic backbone of PBHP-TAPT COF see any appreciable changes upon exposure of the material to $\mathrm{HCl}$ vapours. However, the typical absorption peaks of the triazine units shift in the protonated network from 1501 to $1504 \mathrm{~cm}^{-1}$ (stretching mode), from 1366 to $1362 \mathrm{~cm}^{-1}$ (breathing mode) and from 809 to $811 \mathrm{~cm}^{-1}$ (out-of-plane bending) These miniscule shifts have been reported previously for protonated triazines $32,35,36$. Peak positions are fully recovered after regeneration using $\mathrm{NH}_{3}$ vapours.

Thus, we conclude that the basic triazine moieties are preferentially protonated when the framework is exposed to $\mathrm{HCl}$ vapours. This protonation gives the triazine moieties a localized positive charge, drawing $\pi$-electron density from its neighbouring functional groups ${ }^{23}$. The reversible optical and electronic response of the bulk powder is all-encompassing with no evidence of mixed-phase behaviour, which suggests that $\mathrm{HCl}$ (and later $\mathrm{NH}_{3}$ ) vapours can access the entire latent pore volume. Spontaneous access to latent pore volume can be triggered by local structural changes and has been observed previously for $2 \mathrm{D}$ porous materials that were exposed to strongly interacting guest molecules such as $\mathrm{CO}_{2}$ or iodine in contrast to the physisorption of weakly interacting guests such as $\mathrm{N}_{2}$ and $\mathrm{Ar}^{37,38}$.

We have seen so far that reversible activation of PBHP-TAPT COF by acid vapours not only decreases the optical band gap by $0.3 \mathrm{eV}$, but also attenuates the photoluminescence of the material. (Supplementary Fig. 13 and Supplementary Movie 2). Protonation of the triazine moieties is the most likely explanation for this behaviour, yet there is a composite effect that involves the entire aromatic backbone of PBHP-TAPT COF. Thus, we examine the effect of this reversible chemisorption on the charge-carrier mobility in bulk PBHP-TAPT COF (Supplementary Fig. 23). To date, the most common technique to tune the electrical conductivity of COFs is iodine or sulphur doping ${ }^{39-41}$. However, to the best of our knowledge there are no reports to reversibly tune charge-carrier mobility in COFs in real-time. Pristine PBHP-TAPT COF has a normalised conductivity of $1.32 \times 10^{-8} \mathrm{~S} \mathrm{~m}^{-1}$. On protonation, the conductivity of activated PBHP-TAPT COF increases by 170 -fold to $2.18 \times 10^{-6} \mathrm{~S} \mathrm{~m}^{-1}$ (Fig. 3c). Furthermore, the conductivity drops close to the original value $\left(1.23 \times 10^{-8} \mathrm{~S} \mathrm{~m}^{-1}\right)$ when the sample is regenerated with $\mathrm{NH}_{3}$ vapours. This trend in the overall electrical conductivity is reproduced up to five cycles (Supplementary Fig. 24).

In summary, we report a $2 \mathrm{D}$ COF made up of chemoresistant $\beta$-amino enone bridges (PBHP) and Lewis-basic triazine moieties (TAPT) capable of real-time, reversible optical and electronic sensing of volatile acids and bases. This study shows that optical and electronic activation of PBHP-TAPT COF is achieved by preferential protonation of the triazine nitrogens, which results in an optical response visible to the naked eye and an increase of bulk conductivity by two orders of magnitude. The single-site protonation triggers $\pi$-electron donation into the triazine ring and is accompanied by fluorescence quenching. Both the optical and electronic effects involve the entirety of the $\pi$-aromatic framework and are made possible because PBHP-TAPT COF is not only chemically stable to the corrosive trigger molecules, but it is also permanently porous to the chemisorbed guests. Activation of the framework is fully reversible by chemical triggers $\left(\mathrm{NH}_{3}\right)$, by heating or by vacuum. These findings demonstrate a powerful approach to design more practical sensors and switches, and take genuine advantage of the chemoresistant make-up, of the porous structure, and of the overall conjugation of fully-aromatic donor-acceptor PBHPTAPT COF

\section{Methods}

General. Unless otherwise stated, all materials were commercially available and used without further purification. All solvents were of analytical grade and used without further purification. 4-Aminobenzonitrile, trifluoromethanesulfonic acid (TFMSA) were purchased from Acros Organics. 1,4-Diacetylbenzene, potassium tert-butoxide and ethyl formate were purchased from Sigma-Aldrich.Co. Ltd. (TCI).

Characterization. Powder X-ray (PXRD): measurements were performed with a Bruker D8 Advance diffractometer using Bruker AXS D8 Advanced SWAX diffractometer with $\mathrm{Cu} \mathrm{Ka}(\lambda=0.15406 \mathrm{~nm})$ as a radiation source. Samples were measured from 1 to $80^{\circ} 2 \theta$ with the step of $0.021^{\circ} 2 \theta$ secondary graphite monochromator and LYNXEYE XE detector. Elemental analyses (EA) (C, H and $\mathrm{N})$ : were performed using a PE 2400 Series II CHN Analyzer. Fourier transformed infrared spectroscopy (FT-IR): spectra were recorded on an AVATAR 370 FT-IR 
a

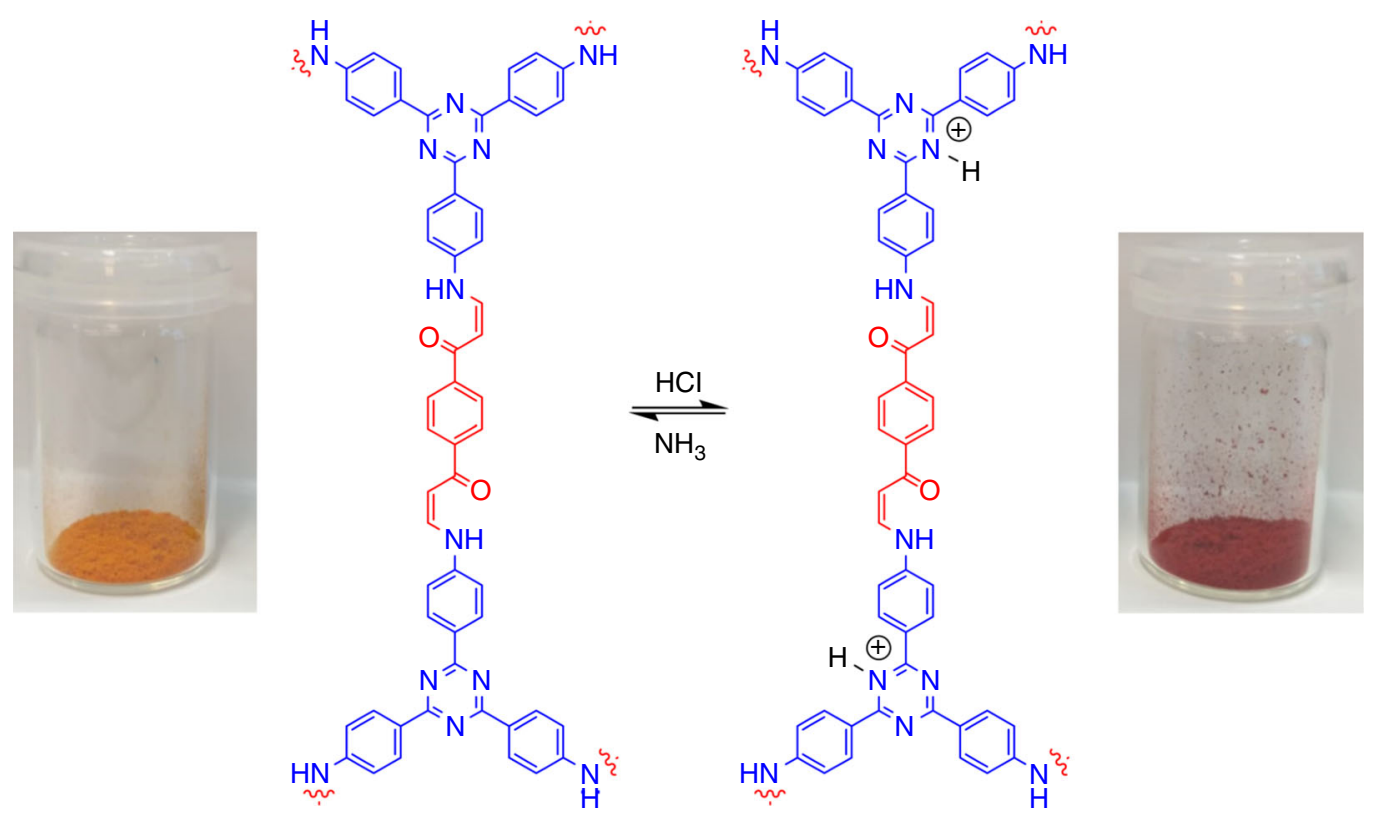

b

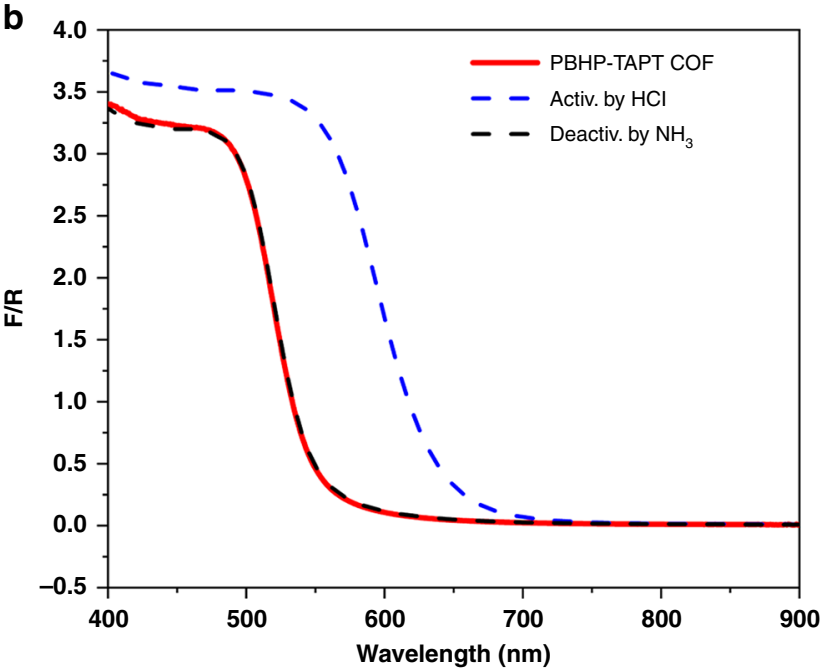

C

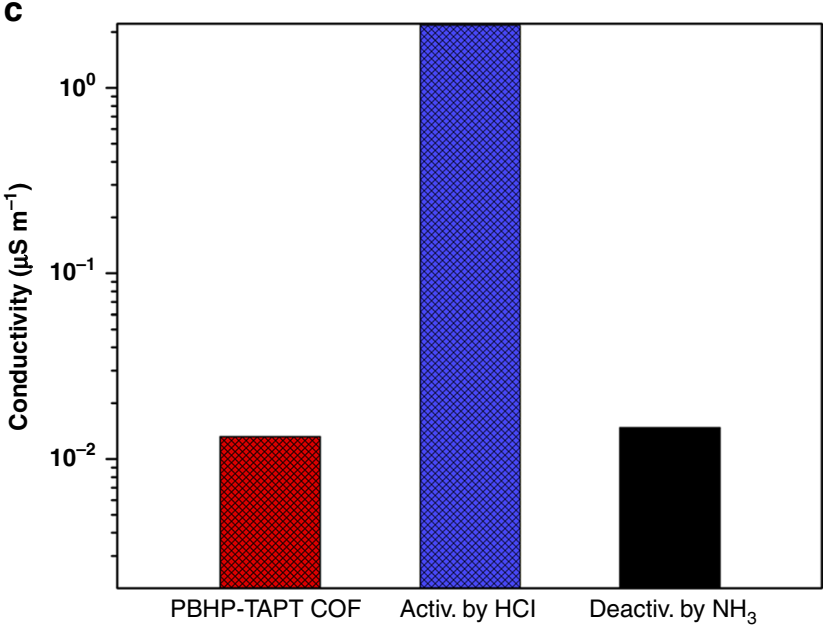

Fig. 3 Opto-electronic response of PBHP-TAPT COF. a Protonation and deprotonation of the triazine moieties with chemical triggers $\left(\mathrm{HCl}\right.$ and $\mathrm{NH}_{3}$ vapours): Pristine, bulk PBHP-TAPT COF orange powder (left) and the activated, red powder, after 2-3 s of exposure to $\mathrm{HCl}$ (right). b Solid-state UV/Vis spectra of pristine PBHP-TAPT COF (red), after activation with $\mathrm{HCl}$ vapours (blue; dashed and dotted line), and regeneration with $\mathrm{NH}_{3}$ vapours (black; dashed line). c Electrical conductivity of pristine PBHP-TAPT COF (red), after activation with $\mathrm{HCl}$ vapours (blue), and regeneration with $\mathrm{NH}_{3}$ vapours (black)

spectrometer from Thermo Nicolet. Solid-state nuclear magnetic resonance (NMR) spectroscopy: ${ }^{13} \mathrm{C}$ CP/MAS spectrum (NMR) of the TAPT-COFs was recorded on Agilent DD2 600 Solid NMR System with $3.2 \mathrm{~mm}$ zirconia rotors. The spinning rate is $13 \mathrm{kHz}$ and the contact time was $10 \mathrm{~ms} .{ }^{1} \mathrm{H}$ and ${ }^{13} \mathrm{C}$ NMR spectra of the monomers were recorded on a Bruker Advance 400 instrument. Chemical shifts $(\delta)$ were reported in ppm. Thermogravimetric analysis (TGA): Thermal gravimetric analysis was carried out on Setsys Evolution 18 thermal analyser from Setaram instrument by heating the samples from 50 to $800^{\circ} \mathrm{C}$ under air or nitrogen atmosphere at a heating rate of $10{ }^{\circ} \mathrm{C} \mathrm{min}-1$. Solid-state $\mathrm{UV} / \mathrm{V}$ is measurements (UV/Vis): were undertaken with a $6000 \mathrm{i} \mathrm{UV}-\mathrm{Vis}-\mathrm{NIR}$ spectrometer from Agilent, and solid-state fluorescence measurements recorded on Fluorolog FL3-22 fluorometer (Horiba-Jobin Yvon). Gas sorption measurements and pore size distributions of COFs (BET): $\mathrm{N}_{2}$ adsorption desorption isotherm was tested on Autosorb iQ instrument and measured using Helium mode. All Samples for nitrogen sorption at $77 \mathrm{~K}$ were activated by degassing in vacuum $\left(2 \times 10^{-5} \mathrm{mbar}\right)$ at $110^{\circ} \mathrm{C}$ for $24 \mathrm{~h}$. The surface area was calculated in the relative pressure $(\mathrm{p} / \mathrm{p} 0)$ range from 0.05 to 0.35 . Pore size distributions were calculated for the adsorption as well as for the desorption branch using QS-DFT and NL-DFT models. Testing process was carried out in liquid nitrogen at $77 \mathrm{~K}$ and the specific surface area of the sample was calculated according to the BET (Brunauer-Emmett-Teller). Scanning electron microscope (SEM): images were obtained with a Nova NanoSEM 450 from FEI. The dry samples were prepared on $15 \mathrm{~mm}$ aluminium stubs using an adhesive, high purity carbon tab. Imaging was conducted at a working distance of $5 \mathrm{~mm}$ and a working voltage of $3-10 \mathrm{kV}$ using a mix of upper and lower secondary electron detectors. The field emission scanning electron microscope measurement scale bar was calibrated against certified standards. Transmission electron microscopy (TEM): was carried out using a The FEI Talos ${ }^{\text {tw }}$ F200X is a $200 \mathrm{kV}$ FEG operating at an accelerating voltage of $200 \mathrm{kV}$ with a spherical aberration coefficient: value of $5.6 \mathrm{~mm}$. Images were recorded on Ceta $^{\text {at }} 16 \mathrm{M}$ camera, which combined with an embedded Piezoenhanced stage. Atomic force microscopy (AFM): measurements were performed with Bruker Multimode VIII equipped with E-Scanner. The PBHP-TAPT COF was suspended in THF and sonicated for $30 \mathrm{~min}$ in an ultrasonicator. Then the supernatant solution was drop casted onto a silicon substrate (with $300 \mathrm{~nm}-\mathrm{SiO}_{2}$ layer). Peak Force Tapping mode was employed with the cantilever SCANASYSTAIR.

Conductivity measurments. Electrical conductivity was determined by two-point I-V measurements. Powder samples were pelletized into a disk between two stainless rods supported by an insulating plastic insert with an inner diameter of 8 $\mathrm{mm}$. I-V profiles were obtained and recorded at RT using a source meter, Keithley 
$2612 \mathrm{~A}$, with the voltage ranging from -10 to $10 \mathrm{~V}$ in typical experiments. Average of minimum three separate measured current values was analysed to calculate the conductivity of each sample.

Computational methods. All the simulations are based on density functional theory and were performed using the projector augmented wave (PAW), formalism within the generalised gradient approximation (GGA) method with Perdew-BurkeErnzerhof (PBE) exchange-correlation functional as implemented in Vienna $\mathrm{Ab}$ Initio Simulation Package (VASP). The cut-off energy of $800 \mathrm{eV}$ for the plane-wave basis set has been consistently used in all calculations. The convergence criterion of 0.01 was used for the forces in geometry optimizations and $10^{-5} \mathrm{eV}$ was used for the energy convergence. The lattice parameters and atomic positions were fully relaxed. The Brillouin zone for structure optimizations was sampled with the Monkhorst-Pack, special k-point mesh on $1 \times 1 \times 3$. D3 dispersion correction was adopted in all calculations. All geometry optimizations were performed with the unit cell consisting of two layers of $\mathrm{COF}$, starting from the geometry described as AA-eclipsed, AA-serrated and AB. The total energies of AA-eclipsed, AA-serrated and $\mathrm{AB}$ are $-2101.53,-2101.43$ and $-2097.23 \mathrm{eV}$, respectively. Both $\mathrm{AA}$ structures are more stable than $\mathrm{AB}$.

\section{Data availability}

All data generated or analysed during this study are provided as a Source Data file at https://doi.org/10.5281/zenodo.3249392 (URL: https://zenodo.org/record/3249392).

Received: 10 February 2019 Accepted: 28 June 2019

Published online: 19 July 2019

\section{References}

1. Ekanayake, E. M., Preethichandra, D. M. \& Kaneto, K. Polypyrrole nanotube array sensor for enhanced adsorption of glucose oxidase in glucose biosensors. Biosens. Bioelectron. 23, 107-113 (2007).

2. Zhao, X., Chaudhry, S. T. \& Mei, J. in Adv. Heterocycl. Chem. Vol. 121, pp. 133-171 (Elsevier, Cambridge, MA, 2017).

3. Ostroverkhova, O. Organic optoelectronic materials: mechanisms and applications. Chem. Rev. 116, 13279-13412 (2016).

4. Gao, X. \& Zhao, Z. High mobility organic semiconductors for field-effect transistors. Sci. China. 58, 947-968 (2015)

5. Christogiannis, N. et al. Characterizing the electroluminescence emission from a strongly coupled organic semiconductor microcavity LED. Adv. Opt. Mater. 1, 503-509 (2013).

6. McGehee, M. D. \& Goh, C. in Frontiers of Engineering: Reports on LeadingEdge Engineering from the 2005 Symposium. (National Academies Press, Washington, DC, 2006).

7. Roncali, J. Molecular engineering of the band gap of $\pi$-conjugated systems: facing technological applications. Macromol. Rapid Commun. 28, 1761-1775 (2007).

8. Kessler, F. K. et al. Functional carbon nitride materials-design strategies for electrochemical devices. Nat. Rev. Mater. 2, 17030 (2017).

9. Irfan, A., Aftab, H. \& Al-Sehemi, A. G. Push-pull effect on the geometries, electronic and optical properties of thiophene based dye-sensitized solar cell materials. J. Saudi Chem. Soc. 18, 914-919 (2014).

10. Ding, S.-Y. \& Wang, W. Covalent organic frameworks (COFs): from design to applications. Chem. Soc. Rev. 42, 548-568 (2013).

11. Zhao, W., Xia, L. \& Liu, X. Covalent organic frameworks (COFs): perspectives of industrialization. CrystEngComm 20, 1613-1634 (2018).

12. Huang, N., Wang, P. \& Jiang, D. Covalent organic frameworks: a materials platform for structural and functional designs. Nat. Rev. Mater. 1, 16068 (2016).

13. Diercks, C. S. \& Yaghi, O. M. The atom, the molecule, and the covalent organic framework. Science 355, eaal1585 (2017).

14. Bisbey, R. P. \& Dichtel, W. R. Covalent organic frameworks as a platform for multidimensional polymerization. ACS Cent. Sci. 3, 533-543 (2017).

15. Matsumoto, M. et al. Rapid, low temperature formation of imine-linked covalent organic frameworks catalyzed by metal triflates. J. Am. Chem. Soc. 139, 4999-5002 (2017).

16. Vitaku, E. \& Dichtel, W. R. Synthesis of $2 \mathrm{D}$ imine-linked covalent organic frameworks through formal transimination reactions. J. Am. Chem. Soc. 139, 12911-12914 (2017).

17. Schwarz, D. et al. Tuning the porosity and photocatalytic performance of triazine-based graphdiyne polymers through polymorphism. ChemSusChem, 19, 194-199 (2018).

18. Kochergin, Y. S. et al. Exploring the "Goldilocks Zone" of semiconducting polymer photocatalysts by donor-acceptor interactions. Angew. Chem. Int. Ed. 57, 14188-14192 (2018).
19. Biswal, B. P. et al. Mechanochemical synthesis of chemically stable isoreticular covalent organic frameworks. J. Am. Chem. Soc. 135, 5328-5331 (2013).

20. Schwarz, D. et al. Twinned growth of metal-free, triazine-based photocatalyst films as mixed-dimensional (2D/3D) van der Waals heterostructures. $A d v$. Mater. 29, 1703399 (2017).

21. Algara-Siller, G. et al. Triazine-based graphitic carbon nitride: a twodimensional semiconductor. Angew. Chem. 126, 7580-7585 (2014).

22. Rao, M. R., Fang, Y., De Feyter, S. \& Perepichka, D. F. Conjugated covalent organic frameworks via michael addition-elimination. J. Am. Chem. Soc. 139, 2421-2427 (2017)

23. Elbe, F. et al. Photochemical and photophysical deactivation of 2, 4, 6-triaryl1, 3, 5-triazines. J. Phys. Chem. A 104, 8296-8306 (2000).

24. Kandambeth, S. et al. Construction of crystalline $2 \mathrm{D}$ covalent organic frameworks with remarkable chemical (acid/base) stability via a combined reversible and irreversible route. J. Am. Chem. Soc. 134, 19524-19527 (2012).

25. Medina, D. D. et al. Room temperature synthesis of covalent-organic framework films through vapor-assisted conversion. J. Am. Chem. Soc. 137, 1016-1019 (2015).

26. Pachfule, P. et al. Diacetylene functionalized covalent organic framework (COF) for photocatalytic hydrogen generation. J. Am. Chem. Soc. 140, 1423-1427 (2018).

27. Clougherty, L., Sousa, J. \& Wyman, G. C $=\mathrm{N}$ stretching frequency in infrared spectra of aromatic azomethines. J. Org. Chem. 22, 462-462 (1957).

28. Waller, P. J. et al. Chemical conversion of linkages in covalent organic frameworks. J. Am. Chem. Soc. 138, 15519-15522 (2016).

29. Niu, F., Tao, L.-M., Deng, Y.-C., Wang, Q.-H. \& Song, W.-G. Phosphorus doped graphene nanosheets for room temperature NH 3 sensing. N. J. Chem. 38, 2269-2272 (2014).

30. Tao, L.-M., Niu, F., Zhang, D., Wang, T.-M. \& Wang, Q.-H. Amorphous covalent triazine frameworks for high performance room temperature ammonia gas sensing. N. J. Chem. 38, 2774-2777 (2014).

31. $\mathrm{Xu}, \mathrm{N}$. et al. Continuous detection of $\mathrm{HCl}$ and $\mathrm{NH} 3$ gases with a highperformance fluorescent polymer sensor. N. J. Chem. 42, 13367-13374 (2018)

32. Du, X., Zou, G., Wang, Z. \& Wang, X. A scalable chemical route to soluble acidified graphitic carbon nitride: an ideal precursor for isolated ultrathin $\mathrm{gC} 3$ N 4 nanosheets. Nanoscale 7, 8701-8706 (2015).

33. Cui, Y. et al. Design and synthesis of a multifunctional porous $\mathrm{N}$-rich polymer containing s-triazine and Tröger's base for CO 2 adsorption, catalysis and sensing. Polym. Chem. 9, 2643-2649 (2018).

34. Welch, G. C. \& Bazan, G. C. Lewis acid adducts of narrow band gap conjugated polymers. J. Am. Chem. Soc. 133, 4632-4644 (2011).

35. Ong, W.-J., Tan, L.-L., Chai, S.-P., Yong, S.-T. \& Mohamed, A. R. Surface charge modification via protonation of graphitic carbon nitride (g-C3N4) for electrostatic self-assembly construction of $2 \mathrm{D} / 2 \mathrm{D}$ reduced graphene oxide (rGO)/g-C3N4 nanostructures toward enhanced photocatalytic reduction of carbon dioxide to methane. Nano Energy 13, 757-770 (2015).

36. Zhang, Y., Thomas, A., Antonietti, M. \& Wang, X. Activation of carbon nitride solids by protonation: morphology changes, enhanced ionic conductivity, and photoconduction experiments. J. Am. Chem. Soc. 131, 50-51 (2008).

37. Chaoui, N., Trunk, M., Dawson, R., Schmidt, J. \& Thomas, A. Trends and challenges for microporous polymers. Chem. Soc. Rev. 46, 3302-3321 (2017).

38. Yao, C., Li, G., Wang, J., Xu, Y. \& Chang, L. Template-free synthesis of porous carbon from triazine based polymers and their use in iodine adsorption and $\mathrm{CO}_{2}$ capture. Sci. Rep. 8, 1867 (2018).

39. Ding, $H$. et al. A tetrathiafulvalene-based electroactive covalent organic framework. Chem. Eur. J. 20, 14614-14618 (2014).

40. Liao, $\mathrm{H}$. et al. A $2 \mathrm{D}$ porous porphyrin-based covalent organic framework for sulfur storage in lithium-sulfur batteries. J. Mater. Chem. A 4, 7416-7421 (2016).

41. Wan, S., Guo, J., Kim, J., Ihee, H. \& Jiang, D. A belt-shaped, blue luminescent, and semiconducting covalent organic framework. Angew. Chem. 120, 8958-8962 (2008)

\section{Acknowledgements}

This work was primarily funded by European Research Council (ERC) for funding under the Starting Grant Scheme (BEGMAT-678462). We thank Adam Malek for access to IR spectroscopy, and Dr. Martin Dracinsky for access to solid-state NMR facilities. Christoph Erdmann and Prof. Nicola Pinna for TEM measurements. Prof. Emil List Kratochvil for the access to their integrating sphere. Prof. Dr. Jürgen P. Rabe for access to AFM measurments and Prof. Christoph T. Koch and Johannes Müller for some additional SEM measurments.

\section{Author contributions}

R.K. carried out the synthetic experiments, analysed the data and wrote the paper. Y.N. carried out the conductivity measurements and analysis. D.K.B. and R.K. synthesised the 
sample compounds and building blocks. Y.S.K. helped with the solid-state UV/Vis and thermogravimetric analysis. B.B performed $\mathrm{N}_{2}$ sorption measurements and analysis. P.L. and P.N. performed the DFT calculations. M.J.B. conceived the experiments and wrote the manuscript.

\section{Additional information}

Supplementary Information accompanies this paper at https://doi.org/10.1038/s41467019-11264-z.

Competing interests: The authors declare no competing interests.

Reprints and permission information is available online at http://npg.nature.com/ reprintsandpermissions/

Peer review information: Nature Communications thanks Xiaomei Zhang and other anonymous reviewer(s) for their contribution to the peer review of this work. Peer reviewer reports are available
Publisher's note: Springer Nature remains neutral with regard to jurisdictional claims in published maps and institutional affiliations.

(c) (i) Open Access This article is licensed under a Creative Commons Attribution 4.0 International License, which permits use, sharing, adaptation, distribution and reproduction in any medium or format, as long as you give appropriate credit to the original author(s) and the source, provide a link to the Creative Commons license, and indicate if changes were made. The images or other third party material in this article are included in the article's Creative Commons license, unless indicated otherwise in a credit line to the material. If material is not included in the article's Creative Commons license and your intended use is not permitted by statutory regulation or exceeds the permitted use, you will need to obtain permission directly from the copyright holder. To view a copy of this license, visit http://creativecommons.org/ licenses/by/4.0/.

(C) The Author(s) 2019 